Questions vives

\section{Questions Vives}

Recherches en éducation

Vol.4 $n^{\circ} 13$ | 2010

Dispositif didactique ? Dispositif pédagogique?

Situations d'apprentissage !

\title{
Rencontre avec Stéphane Simonian à propos de son ouvrage « Hypertexte et processus cognitifs, quels enjeux pour l'apprentissage »
}

\section{Stéphane Simonian}

\section{(2) OpenEdition}

\section{Journals}

\section{Édition électronique}

URL : http://journals.openedition.org/questionsvives/339

DOI : 10.4000/questionsvives.339

ISBN : 978-2-8218-1086-0

ISSN : $1775-433 \mathrm{X}$

Éditeur

Université Aix-Marseille (AMU)

\section{Édition imprimée}

Date de publication : 1 janvier 2010

Pagination : 401-409

ISBN : 978-2-912643-37-7

ISSN : 1635-4079

Référence électronique

Stéphane Simonian, «Rencontre avec Stéphane Simonian à propos de son ouvrage " Hypertexte et processus cognitifs, quels enjeux pour l'apprentissage » », Questions Vives [En ligne], Vol. $4 n^{\circ} 13$ | 2010, mis en ligne le 01 juin 2011, consulté le 23 avril 2019. URL : http://journals.openedition.org/ questionsvives/339; DOI : 10.4000/questionsvives.339

\section{(c) $(1)$}

Questions Vives est mis à disposition selon les termes de la licence Creative Commons Attribution -

Pas d'Utilisation Commerciale - Pas de Modification 4.0 International. 


\section{ENTRETIEN}

\section{Rencontre avec Stéphane Simonian à propos de son ouvrage " Hypertexte et processus cognitifs, quels enjeux pour l'apprentissage?"}

Stéphane Simonian vient de publier chez Lavoisier un ouvrage intitulé Hypertexte et processus cognitifs, quels enjeux pour l'apprentissage ? Cette publication, partiellement issue de sa thèse, est enrichie par des compléments originaux notamment en matière de scénarisation dans l'enseignement à distance par Internet.

Simonian, S. (2010). Hypertexte et processus cognitifs, quels enjeux pour l'apprentissage? Paris : Lavoisier, 259p.

Introduction.

Chapitre 1. Hypertexte : définitions, caractéristiques et premiers enjeux.

Chapitre 2. Hypertexte : intérêts et limites des recherches en sciences cognitives pour l'apprentissage.

Chapitre 3. Modèles pédagogiques appliqués aux machines à enseigner et applicables aux environnements informatiques.

Chapitre 4. Transposition des savoirs et appropriation des savoirs via les hypertextes. Chapitre 5. Principes pour concevoir un scénario pédagogique. Conclusion. Bibliographie. Index.

[www.lavoisier.fr] 


\section{Rencontre avec Stéphane Simonian à propos de son ouvrage "Hypertexte et processus cognitifs, quels enjeux pour l'apprentissage "}

Questions Vives, Recherches en Education : L'étude des dispositifs hypermédias et celle de la relation homme-machine ont fait l'objet de nombreuses publications entre 1990 et 2000. Toutefois vous semblez désirer reprendre cette thématique sous un angle renouvelé. En quoi "l'apprenaute", seul capable de donner du sens à ce qu'il cherche rend-il possible un changement de perspective?

Stéphane Simonian : D'une manière générale nous voyons que le réseau internet en tant qu'ensemble de réseaux documentaires, mais aussi de réseaux sociaux, dépend principalement de l'initiative de l'internaute. C'est cette dernière qui permet au réseau de se construire comme de se déconstruire. Le concept d'apprenaute se réfère au fait que l'internaute subit peu de contraintes et bénéficie d'instruments puissants en matière d'apprentissage. Cela est vrai sur le plan matériel, la souris en est un exemple car elle permet de "donner la main " à l'internaute, mais aussi logiciel, comme par exemple les moteurs de recherche ou l'hypertexte.

De manière plus spécifique, si l'apprenaute est bien au centre des dispositifs informatiques d'apprentissage, deux approches semblent à distinguer. La première est celle qui concerne l'exploration, la découverte ou encore la «navigation» au sein des environnements informatiques peu contraints mais guidant l'apprenaute dans sa manière de se construire des connaissances. L'idiosyncrasie en est un principe fondamental. Les hypermédias sont alors au service de l'apprenaute dans un apprentissage basé sur la "découverte ", pour reprendre les propos de Tricot, et peu sur un "apprentissage par instruction ". L'idée d'apprenaute permet ainsi d'indiquer que nous transitons du modèle de l'instruction vers des modèles de l'apprentissage. La deuxième approche est liée à la mise en avant de modèles descriptifs dépendant de l'usage plutôt que de modèles prescriptifs. Ainsi, les hypermédias, bien que dépendant des concepteurs, prennent du sens, trouvent une utilité, en fonction des usages et non exclusivement en fonction d'un modèle prescrit.

II est donc important d'étudier l'affordance des hypermédias. II semble cependant que si l'informatique est un instrument de contrôle et de traçage, les données informatiques fournies par le système sont encore loin d'être suffisantes pour comprendre ce qu'un apprenaute comprend, apprend, au sein des environnements informatiques d'apprentissage. II est alors nécessaire de se focaliser sur les interactions à distance, objet de plus en plus étudié de nos jours. Mais bien plus que cela, il s'agit de changer notre regard sur les hypermédias en ne les considérant plus comme des instruments d'automatisation des tâches mais comme des instruments d'accompagnement dans la réalisation de tâches ou d'activités d'apprentissage, comme la réalisation d'une étude de cas, la résolution d'une situation problème, etc.

Ainsi, je ne prétends pas renouveler la problématique des hypermédias mais plutôt mettre l'accent, en m'appuyant sur des références historiques et en psychologie cognitive, sur la nécessité de considérer les hypermédias comme répondant à un besoin initié par des utilisateurs dans leurs explorations. C'est ce qu'avaient imaginé les pionniers que sont Paul Otlet et Vanevar Bush qui cherchaient à faciliter l'accès, la trouvaille et la circulation 


\author{
Entretien avec Stéphane Simonian \\ à propos de son ouvrage Hypertexte et processus cognitifs
}

d'informations et de savoirs. Si la dématérialisation des supports a été pensée au cours des années 1930, il en est de même des accès différenciés pour atteindre une même information, de l'encyclopédie collective.

Enfin, du point de vue de la recherche, il est en effet surprenant de constater le manque de preuve de l'efficacité des hypermédias sur les processus cognitifs alors que précisément les outils que sont l'hypertexte, les frames, les layers, etc., sont en lien avec des recherches menées en sciences cognitives. II me semble donc important de continuer à investiguer tout cela et, en recourant au concept d'apprenaute, de déplacer la focalisation des recherches des instruments vers les hommes, des habiletés vers les processus.

L'utilité de l'hypertexte est bien liée à des actions ou des parcours intentionnels lors de la recherche d'une information sur le réseau Internet, par exemple. II est alors opportun d'étudier la dynamique de la consultation plutôt que son résultat (qui consiste à la trouvaille ou non de l'information, sa localisation, etc.). A ce titre, une recherche en cours tend à montrer que les parcours pour un public homogène sont souvent les mêmes jusqu'à ce que les utilisateurs acquièrent des connaissances, rendant les recherches, ainsi que les trouvailles qui en découlent, plus spécifiques. Ceci implique aussi des positionnements méthodologiques particuliers pertinents à l'objet de recherche: approche expérimentale, différentielle, anthropologique ou encore systémique. Ceci questionne notre discipline, les sciences de l'éducation, dans sa manière d'étudier l'être humain dans une situation d'apprentissage. Les hypermédias peuvent être considérés comme une opportunité pour étudier les processus de construction des connaissances (cognitifs et métacognitif) au sein d'une situation d'apprentissage.

QVRE : Vous proposez de passer d'une approche centrée sur " l'individuel » pour vous centrer sur le «personnel ». Est-ce que cela recoupe votre définition de l'apprenaute?

SS : En effet. Un principe qui me semble fort important est de pouvoir considérer les apprenants comme des personnes et non comme individus. Nous retrouvons là un relativisme culturel, proche des vues de Montaigne, où il serait possible de regrouper une certaine diversité de personnes si nous savons quelle culture les relie. Le réseau Internet en est un exemple saisissant de cette reconfiguration culturelle à partir du moment où il est considéré comme un réseau sémantique.

$\mathrm{Si}$ nous nous situons dans un environnement informatique d'apprentissage, nous comprenons qu'il est nécessaire que cet environnement soit assez " ouvert » pour permettre de tenir compte de différentes approches de l'apprenaute, par exemple pour trouver une ressource, ou répondre à une situation-problème. Ainsi, une des caractéristiques est de pouvoir tenir compte de la diversité des apprenautes sans mettre en place un dispositif de type "enseignement assisté par ordinateur " avec des apprentissages par niveau via des pré-tests puis des post-tests. Nous pouvons alors tenir compte des "connaissances préalables " sans prévoir un parcours établi en fonction d'un niveau initial. Si l'environnement est bien conçu, l'apprenaute pourra naviguer à sa guise. II ne s'agit pas de faire des parcours-programmes mais des parcours-guides qui offrent la possibilité d'avancer de manière non linéaire, où des repères sont fournis plutôt que des étapes. Considérer l'apprenant comme un apprenaute, c'est le prendre comme une personne à part entière ayant la possibilité d'emprunter un parcours qui lui convient, sachant que ceux qui lui sont proposés sont pensés dans une perspective d'apprentissage. II n'y a donc plus de bon ou de mauvais 
Dispositif pédagogique ? Dispositif didactique? Situations d'apprentissage !

parcours mais un cheminement qui fait sens pour une personne et qui peut être lié à un contexte particulier, comme la sémantique utilisée par exemple. Par analogie, nous pourrions dire que personnaliser l'apprentissage c'est considérer qu'une note de musique est une expression du corps pour atteindre nos âmes.

QVRE : A la lecture de votre texte, on sent la volonté d'articuler une problématique d'ordre documentaire avec une seconde plus centrée sur le cheminement de la pensée. Vous semblez réfuter ce déterminisme qui fait dire à certains, comme Barbara Cassin par exemple, que certains dispositifs, comme Google, finissent par penser pour nous. Qu'en est-il selon vous?

SS : Je suis plutôt d'avis que les objets informatiques comme Google sont simplement des outils qui nous " aident " à penser. Mais je ne nie pas qu'il existe une "sérendipidité " qui fait que parfois nous trouvons une information que nous n'envisagions pas trouver au départ. Le débat entre une technologie qui nous aide à penser et une technologie qui penserait à notre place est assez vif. La frontière entre les deux semble fragile. Si nous prenons l'exemple d'un moteur de recherche tel que Google, c'est bien l'internaute qui choisit ses propres mots-clefs, puis qui clique sur les liens qui lui semblent pertinents. Mais d'un autre côté, nous pouvons remarquer que certains liens sont toujours utilisés ou activés du fait de leur "popularité ", au détriment d'autres, moins visibles, qui sont portant peut-être plus pertinents.

Nous pouvons nous demander aussi si nous n'oublions pas que les moteurs de recherche et l'hypertexte sollicitent une certaine métacognition. En effet, peu d'utilisateurs sont formés ou savent se questionner sur ce qu'ils cherchent et comment ils cherchent. Nous étudions trop l'efficacité en matière de recherche d'information (pages consultées, nombre de clics sur une page, etc.) et trop peu la dynamique qu'elle engendre. Ainsi, il s'agit de différencier l'action de "naviguer » de l'acte de rechercher. Un autre élément à considérer est le fait que le réseau Internet est un réseau sémantique c'est-à-dire que les liens de pertinence proposés sont aussi des liens produits par l'action collective. A chaque fois que nous activons un lien hypertexte nous participons donc à la construction d'un réseau sémantique en indiquant que pour nous ce lien apparaît a priori pertinent. Nous renforçons la pertinence de ce lien comme des milliers d'autres internautes, même si cette pertinence reste discutable dans la mesure où nous ne trouvons pas forcément l'information recherchée suite à l'activation du lien.

D'une manière plus générale, il me semble que ce débat résume l'opposition classique entre l'homme et la machine. La machine automatise, hiérarchise et nous pourrions dire qu'elle détermine des parcours, des manières de penser en imposant par exemple des liens de pertinence. Cela a été notamment le cas avec les «tuteurs " dits "intelligents ». Mais rappelons qu'un des principes du fondement du réseau Internet, mais aussi du lien hypertexte encore d'actualité, est lié à une récursivité entre l'homme et la machine. La machine propose, mais l'homme donne du sens en activant un lien hypertexte. Cet exemple 


\author{
Entretien avec Stéphane Simonian \\ à propos de son ouvrage Hypertexte et processus cognitifs
}

nous rappelle que la machine est avant tout animée par un programme même s'il est de plus en plus évolué, modifiable et adaptable aux situations comme la technologie " Androïd" ".

Ces évolutions peuvent inquiéter, notamment en éveillant certains vieux fantasmes propres aux technologies (" elles savent "; " elles pensent »). Mais comme le disait Einstein "les machines un jour pourront résoudre tous les problèmes, mais jamais aucune d'entre elles ne pourra en poser un ". Ou, pour reprendre les propos d'Albert Jacquard, " on peut apprendre à un ordinateur à dire : je t'aime, mais on ne peut pas lui apprendre à aimer ”. Ces différences fondamentales font qu'il ne peut y avoir de déterminisme compris comme un phénomène inéluctable conditionnant l'être humain dans sa manière de penser, à moins que nous considérions que nous sommes semblables à des machines. Si ce qui nous aide à penser transforme à tel point notre manière de penser que nous lirons notre pensée sur notre écran, cela signifie-t-il que nous ne pouvons plus nous questionner sur cette pensée ou douter de ce que nous pensons?

L'idée d'une technologie qui penserait à notre place a été envisagée par les travaux sur l'intelligence artificielle qui, à bien des égards, a mis en évidence qu'il ne peut en être ainsi. Le test de Turing non réussi à ce jour en est un exemple. Le déterminisme dans une approche philosophique ne me semble pas dépendant de la technologie mais plutôt de l'humain. Le débat me semble plutôt ontologique.

QVRE : Pour être "à la hauteur de nos drôles de machines ", comme le déclare Geneviève Jacquinot-Delaunay (p.52), il faut, dites-vous (p.60), disposer d'une grande culture et donc de connaissances antérieures. N'y a-t-il pas là une forme de sélection qui tend à exclure de l'exploitation des liens hypertextuels les usagers qui ne possèdent pas une culture suffisante.

SS : Ceci semble être le cas lors d'une recherche d'information sur le réseau Internet et non au sein d'un environnement d'apprentissage qui doit tenir compte du public apprenant et où des tuteurs sont présents pour accompagner les apprenautes.

Concernant le réseau Internet, et aux vu de recherches récentes, il semble que ce réseau profite à des personnes ayant des connaissances élevées dans le domaine lié à leur expertise. Ainsi, Internet serait en quelque sorte " élitiste ». Mais ne serait-ce pas le point de départ de toute technologie, comme le précisait Bruner. La technologie serait dans un premier temps élitiste avant de se démocratiser. Mais, outre ce point de vue qui peut être considéré comme un a priori, il semble que ce n'est pas uniquement l'usage de l'hypertexte qui est concerné, mais l'exploitation du texte " caché " ou «masqué " par le lien hypertexte. Des connaissances antérieures facilitent alors la trouvaille de l'information recherchée, sa compréhension et son exploitation. Cet ensemble met en évidence la nécessité de former et d'accompagner les internautes afin de réduire les inégalités dans l'accès au savoir. A ce jour, il est difficile d'imaginer un tutorat ou une interaction qui pourrait s'exercer simultanément à la recherche d'information sur le réseau Internet. Mais des outils spécifiques séparés existent tel que les forums.

\footnotetext{
1 Androïd est un système d'exploitation fondé sur Linux, développé par une société rachetée par Google. Ce système est présent sur de récents téléphones mobiles (smartphones) et appareils informatiques nomades et permet d'exécuter des applications logicielles.
} 


\section{Dispositif pédagogique ? Dispositif didactique? Situations d'apprentissage !}

Au sein des environnements informatiques d'apprentissage, il n'en va pas de même car les apprenautes ne sont pas dans des environnements complètement ouverts comme le réseau Internet mais dans des parcours guidés relevant d'un " réseau partiel ". De plus, un interlocuteur est souvent disponible, prenant la fonction de tuteur ou d'animateur. Un des rôles du tuteur, comme d'un enseignant, est bien de veiller à l'égalité dans l'accès et la transmission des savoirs. A travers cette question, nous voyons donc tout l'intérêt pour les Sciences de l'éducation de se saisir de cette question qui concerne les pratiques pédagogiques.

QVRE : Les aspects didactiques sont souvent discutés dans votre ouvrage. Peut-on envisager une "hyperdidactique" qui pourrait convaincre les professionnels de l'enseignement d'intégrer les technologies dans leurs pratiques d'enseignants.

SS : Il est vrai que les aspects didactiques sont souvent cités et discutés dans mon livre. D'une part, parce que de nombreux « principes " didactiques sont appliqués, tel que ceux de la granularité du savoir, la linéarité du savoir, etc., et il faut dire que les technologies sont propices à la formalisation de ces principes didactiques, notamment à travers les scénarios mis en œuvre. Et, d'autre part, parce qu'un paradoxe a attiré mon attention : si la transmission des savoirs semble facilitée par la linéarité, l'appropriation des savoirs, elle, serait non-linéaire. II m'a donc semblé opportun de questionner ce point grâce à des recherches sur l'hypertexte qui permet ces transpositions non linéaires. Si les résultats liés à la non-linéarité ne sont pas probants, cela s'explique surtout par le fait que les variables étudiées sont des habiletés (des performances, par exemple) et laissent de côté la dynamique des processus d'apprentissage mis en œuvre.

Une «hyperdidactique " serait alors liée à la formalisation d'un scénario pédagogique visant l'étude des dynamiques d'apprentissage. De ce point de vue, les environnements informatiques d'apprentissage permettent d'accompagner la dynamique d'apprentissage et sont au service des professionnels de l'enseignement, non pour déléguer l'enseignement à la machine mais pour leur permettre d'enrichir des situations d'apprentissage, et d'accompagner les apprenautes de manière plus personnelle. Pour étudier cela, les interactions asynchrones qui ont lieu sur les forums de discussion permettent au chercheur de recueillir des données de manière dynamique et longitudinale sur les processus mis en $œ u v r e$ et sur leur évolution. Des recherches pourraient porter par exemple sur les phases de compréhension, sur les changements de point de vue sur un objet de savoir...

Cependant "l'hyperdidactisation », notion à laquelle je n'avais pas pensé, nécessite de fournir des éléments concrets aux professionnels de l'enseignement et de la formation pour qu'ils puissent exploiter au mieux les scénarios d'apprentissage. A la fin de l'ouvrage, je propose un tableau synthétique pour formaliser des scénarios d'apprentissage. Peut être ceci participe-t-il d'une forme "d'hyperdidactisation »? Mais, l'essentiel, est bien de considérer que si nous voulons prouver les multiples intérêts que peuvent revêtir les environnements informatiques d'apprentissage, il est nécessaire de les considérer comme des outils d'accompagnement plutôt que des exerciseurs. Nous voyons, par exemple, que les logiciels ludo-éducatifs sont souvent des exerciseurs et ne changent pas de paradigme. II est aussi nécessaire de fournir les moyens d'analyser les dynamiques liées aux processus mis en œuvre. Ce travail de recherche est tout à fait passionnant. 
Entretien avec Stéphane Simonian

à propos de son ouvrage Hypertexte et processus cognitifs

QVRE : Vos préconisations en matière de scénarisation semblent ambitieuses. Au fond vous "faites feu de tout bois " en proposant d'articuler apprentissage par instruction, apprentissage par l'action et apprentissage par la découverte. N'est-ce pas contradictoire avec le fait que les enseignants sont plutôt en phase avec l'une ou l'autre de ces approches et rarement avec toutes ?

SS : Je ne saurais dire si cela est ambitieux. L'idée est de pouvoir scénariser différents types d'apprentissage selon les étudiants, la formation ou la discipline enseignée. II ne s'agit pas d'indiquer qu'un scénario est préférable à un autre mais d'être « logique " par rapport au scénario proposé. Par exemple, si vous préconisez un scénario linéaire basé sur un apprentissage par instruction qui s'apparente à un cours magistral, il semble clair que la parole est monopolisée par l'enseignant représentée par un contenu fortement structuré. II ne faut pas alors être surpris du faible usage des outils de communication proposés et des interactions peu nombreuses. Cependant, ce qui est mis en avant est l'apprentissage par la découverte, car il semble particulièrement adapté aux technologies actuelles. En effet, ce type d'apprentissage repose sur l'exploration, la navigation, les frayages. II semble donc davantage adapté à la potentialité des technologies sachant qu'un apprentissage par la découverte nécessite une forte médiation humaine pour accompagner l'apprenaute, le guider. Dans ce type d'apprentissage il devient nécessaire de formaliser un scénario d'encadrement.

Enfin, ces approches ne sont pas contradictoires mais complémentaires. En effet, au cours d'un enseignement, nous pouvons privilégier des temps, ou des séances, où l'apprentissage est plutôt de type apprentissage par instruction, puis d'autres séances où il s'agit d'appliquer une notion ou une formule ce qui nous rapproche de l'apprentissage par l'action; il est aussi possible de prévoir d'autres séances plus axées sur la découverte. Chacun des scénarios propose d'accompagner l'apprenant à la fois par la structure d'un corpus de savoirs et par le tutorat proposé, encore appelé « scénario d'encadrement ». C'est pour cela que le réseau Internet n'est pas un réseau d'apprentissage mais bien un réseau d'information. Un réseau d'apprentissage est un environnement contraint, et pas uniquement contraignant, qui guide l'apprenant. Le scénario est la formalisation de ce guidage.

QVRE : Quels sont vos projets pour l'avenir?

SS : Mes projets sont triples. Premièrement, continuer à travailler la relation entre l'hypertexte et les processus d'apprentissage d'un point de vue dynamique. Un travail en cours est engagé. Deuxièmement, continuer à étudier la mise en œuvre de scénarios pédagogiques notamment pour fournir aux professionnels de l'éducation et de la formation des méthodes pratiques d'intégration des technologies dans leurs enseignements. Troisièmement, étudier la dynamique d'appropriation des technologies par les professionnels de l'éducation et de la formation sous l'angle de la professionnalisation. Pour ces trois projets de recherche, j'ai la chance de pouvoir collaborer avec d'autres chercheurs spécialisés sur la professionnalisation ou sur la scénarisation. II existe donc des projets que nous pouvons mener seuls et d'autres de manière collaborative.

II en est de même dans une situation d'apprentissage en ligne où nous conjuguons apprentissage solitaire et collectif. Finalement l'internaute est à l'image du chercheur, il explore et est à l'initiative de son exploration. Puis, vient le moment du partage et des rencontres. Ceci est l'objet de l'introduction de l'ouvrage : sur le chemin nous pensons être 
Dispositif pédagogique? Dispositif didactique?

Situations d'apprentissage!

seuls mais il n'en est pas ainsi. Et lorsque nous nous sentons égarés, nous nous arrêtons pour cheminer dans nos pensées afin de prendre une décision. Cette décision peut venir de nous mais aussi d'une autre personne qui peut nous éclairer, nous guider. Même si cette personne ne suit pas le même itinéraire que nous, elle peut comprendre notre cheminement pour mieux nous indiquer la direction à prendre. Là est la différence entre guider et diriger mais aussi entre un modèle prescriptif et descriptif. Tout dépend de la liberté conférée à l'apprenaute mais aussi de la confiance que nous avons en lui.

Simonian, S. (2010). Hypertexte et processus cognitifs, quels enjeux pour l'apprentissage? Paris : Lavoisier, 259p. 